\title{
Molecular guided therapy for advanced pancreatic cancer patients with PI3K activated mutation: vision or illusion?
}

This article was published in the following Dove Press journal:

OncoTargets and Therapy

19 February 2013

Number of times this article has been viewed

\author{
Anas Gazzah' \\ Daniel Barrios Gonzales' \\ Antonin Levy' \\ Rastislav Bahleda' \\ Michel Ducreux ${ }^{2}$ \\ Ludovic Lacroix ${ }^{3}$ \\ Jean Charles Soria' \\ 'SITEP (Service des Innovations \\ Therapeutiques Précoces), \\ Department of Medicine, Institut \\ Gustave Roussy, Paris XI University, \\ Villejuif, France; ${ }^{2}$ Department of \\ Medicine, Institut Gustave Roussy, \\ Paris XI University, Villejuif, France; \\ ${ }^{3}$ Department of Biology, Institut \\ Gustave Roussy, Paris XI University, \\ Villejuif, France
}

\begin{abstract}
Despite a modern validated regimen of chemotherapy, advanced pancreatic adenocarcinoma remains the fourth most common cause of cancer-related death worldwide. The phosphoinositide 3-kinase pathway (PI3K)/Akt/mammalian target of rapamycin (mTOR) is a major signaling pathway that may be activated in advanced pancreatic cancer. To highlight the potential interest of this targetable pathway in selected advanced pancreatic cancer patients, we report herein a patient with an activated PI3K mutation who was treated in a phase I trial evaluating a treatment combination including an mTOR inhibitor.
\end{abstract}

Keywords: pancreatic cancer, PI3K, targeted therapy, molecular profiling

Characterized by a rapid disease progression, a highly invasive tumor phenotype, and resistance to chemotherapy, advanced pancreatic adenocarcinoma (stage III to IV) is the fourth most common cause of cancer-related death worldwide. Although the oxaliplatin and irinotecan plus fluorouracil and leucovorin (FOLFIRINOX) trial demonstrated an improved overall survival to 11.1 months as compared with gemcitabine as first-line therapy in selected patients with metastatic pancreatic cancer, new therapeutic options are still needed. ${ }^{1}$ The phosphoinositide 3-kinase pathway (PI3K)/Akt/mammalian target of rapamycin (mTOR) is a major signaling pathway involved in various solid tumors that may also be activated in advanced pancreatic cancer. ${ }^{2}$ To highlight the potential interest of this targetable pathway in selected advanced pancreatic cancer patients, we report herein a patient with an activated PI3K mutation who was treated in a phase I trial evaluating a combination including an mTOR inhibitor.

A 66-year old man consulted for abdominal pain in December 2007. He had a past medical history of prostatic adenocarcinoma treated by endoscopic resection and radiotherapy (70 Gy/35 fractions in December 2006), duodenal ulcer, asthma, and hypertension. The abdominal CT scan demonstrated extensive liver involvement and a pancreatic mass. The carbohydrate antigen 19.9 (CA19.9) level was augmented at 2000 $\mathrm{UI} / \mathrm{mL}$ and the liver biopsy confirmed the diagnosis of a stage IV (T4N1M1) advanced pancreatic cancer (immunohistochemical staining positive for cytokeratin 7 and 19, and negative for cytokeratin 17 and chromogranin A). The patient subsequently underwent treatment consisting of gemcitabine/erlotinib and then an oral pan-HER inhibitor and an anti-insulin-like growth factor 1 monoclonal humanized antibody. Because of a hepatic progression, the treatment was stopped in January 2010.

A molecular portrait evaluating 48 different genes was performed using the initial liver biopsy and revealed a PI3K activating mutation on exon 10 
(p.Glu545Ala or p.E545A) and a rare activating mutation on exon 27 of NOTCH (p.Cys1692Tyr or p.C1692Y). No epidermal growth factor receptor or KRAS mutation was retrieved. Based on these results, in February 2010, the patient was included in a phase I trial that evaluated neratinib (HKI-272, pan-HER tyrosine kinase inhibitor, $200 \mathrm{mg}$ orally daily) in combination with temsirolimus (mTOR inhibitor, $15 \mathrm{mg}$ intravenously weekly). After two weeks, while the patient had a rapid decrease of the CA19.9 level (Figure 1), the combined therapy was associated with a significant digestive and urinary radiation recall syndrome. Radiation recall was confirmed by rectosigmoidoscopy (lower rectal telangiectasia and chronic ulceration of the anterior anorectal junction), cystoscopy (obstructed bladder), and review of radiotherapy portals (lesions within the irradiated area). Discontinuation of the treatment for 10 days improved the symptoms but adverse effects reappeared (diarrhea, dysuria, and anal pain) after reintroduction of treatments at lower doses. The patient was withdrawn from the trial in March 2010 and he expired 1 month later due to disease progression. No imaging was performed after the initiation of combination chemotherapy with neratinib and temsirolimus. No symptoms or concurrent medications (except for valsartan and omeprazole) related to the patient's past medical history (duodenal ulcer, asthma, and hypertension) were present prior to or during the phase I treatment.

In advanced pancreatic cancer, activating a mutation of a tumor suppressor gene such as KRAS may lead to aberrant activated-cell proliferation pathways including the PI3K/ Akt/mTOR signaling. It is estimated that nearly half of all advanced pancreatic cancer patients have an activated PI3K/ $\mathrm{Akt} / \mathrm{mTOR}$ pathway that correlates with a poorer outcome. ${ }^{2,3}$
Temsirolimus and everolimus, the second most common mTOR inhibitor, were assessed in phase II trials but showed a poor activity in non-selected gemcitabineresistant advanced pancreatic cancer patients. ${ }^{3,4}$ However, Garrido et al reported that everolimus-sensitive pancreatic xenograft tumors were characterized by gene copy number variations and overexpression of genes leading to activation of the $\mathrm{PI} 3 \mathrm{~K} / \mathrm{Akt} / \mathrm{mTOR}$ in comparison to resistant tumours. $^{2}$

Nowadays, molecular profiling has been used to define cancer intrinsic subtypes that may predict clinical outcomes and responses to therapy. In our case that harbored a PI3K mutation, the rapid CA19.9 decrease suggested an early response to the molecular-guided mTOR therapy. Nevertheless, given the early side-effects and wish from the patient to enter palliative care, the tumor response was not confirmed by an adequate imaging evaluation. The exact pathophysiological mechanism of radiation recall is unknown and vascular; epithelial stem-cell inadequacy and a drug hypersensitivity reaction have been evoked..$^{5}$ Radiation recall is a rare event but has yet been described with mTOR inhibitors. ${ }^{6}$ It is unlikely that pre-existing conditions and other prior or concurrent medications have contributed to adverse effects that limited the continuation of the treatment in the patient. Anyway, another team successfully used everolimus to achieve a partial remission in a patient with advanced pancreatic cancer that was induced by Peutz-Jeghers, a syndrome caused by an mTOR tumor-suppressor gene (STK11/LKB1). ${ }^{7}$ Finally, it has been shown that NOTCH modulates the Akt pathway through regulation of the phosphatase and tensin homolog (PTEN) phosphorylation. ${ }^{8}$ It is likely that our patient, as other double NOTCH-PI3K mutated advanced pancreatic

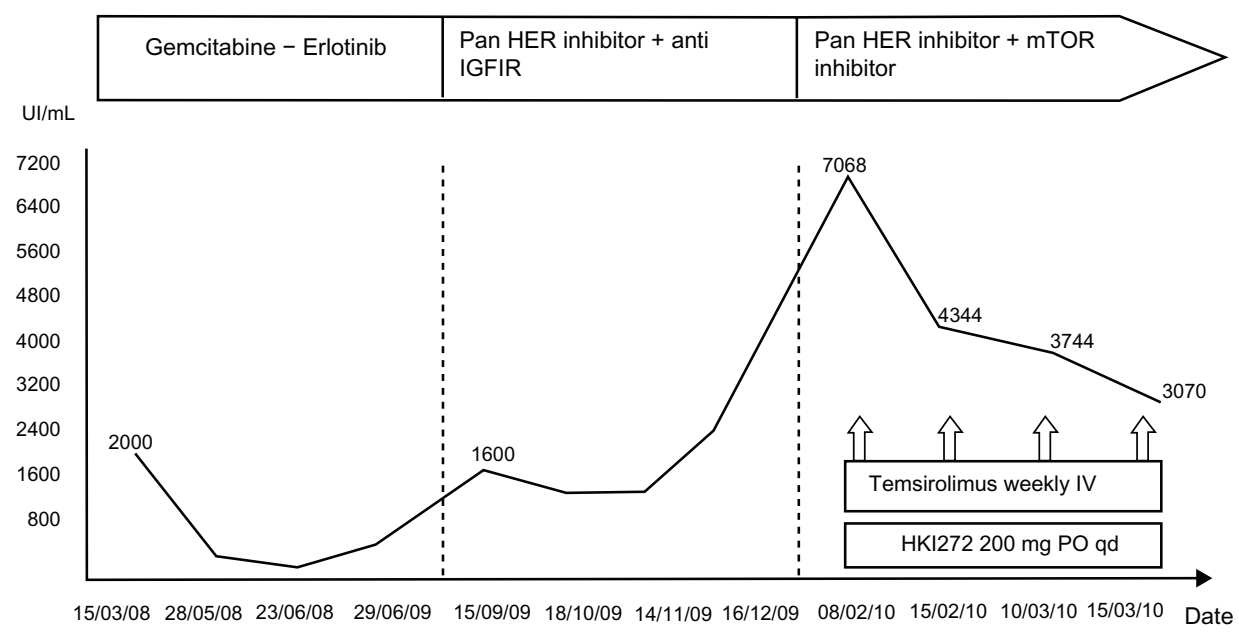

Figure I Tumor marker (CA19.9) evolution during therapy.

Abbreviations: IGFRI, insulin-like growth factor I; mTOR, mammalian target of rapamycin; IV, intravenous; PO, per os; qd, once a day. 
cancer patients, may have derived a clinical benefit from such a combined targeted molecularly driven strategy. Prospective trials are still needed to assess the true efficacy/toxicity ratio of this promising molecularly driven combination.

\section{Disclosure}

The authors have no conflicts of interest to disclose.

\section{References}

1. Conroy T, Desseigne F, Ychou M, et al. FOLFIRINOX versus gemcitabine for metastatic pancreatic cancer. $N$ Engl J Med. 2011; 364(19):1817-1825.

2. Garrido-Laguna I, Tan AC, Uson M, et al. Integrated preclinical and clinical development of mTOR inhibitors in pancreatic cancer. $\mathrm{Br} J$ Cancer. 2010;103(5):649-655.

3. Javle MM, Shroff RT, Xiong H, et al. Inhibition of the mammalian target of rapamycin (mTOR) in advanced pancreatic cancer: results of two phase II studies. BMC Cancer. 2010;10:368.
4. Wolpin BM, Hezel AF, Abrams T, et al. Oral mTOR inhibitor everolimus in patients with gemcitabine-refractory metastatic pancreatic cancer. J Clin Oncol. 2009;27(2):193-198.

5. Camidge R, Price A. Characterizing the phenomenon of radiation recall dermatitis. Radiother Oncol. 2001;59(3):237-245.

6. Bourgier C, Massard C, Moldovan C, Soria JC, Deutsch E. Total recall of radiotherapy with mTOR inhibitors: a novel and potentially frequent side-effect? Ann Oncol. 2011;22(2):485-486.

7. Klümpen HJ, Queiroz KC, Spek CA, et al. mTOR inhibitor treatment of pancreatic cancer in a patient With Peutz-Jeghers syndrome. J Clin Oncol. 2011;29(6):e150-e153.

8. Vo K, Amarasinghe B, Washington K, Gonzalez A, Berlin J, Dang TP. Targeting notch pathway enhances rapamycin antitumor activity in pancreas cancers through PTEN phosphorylation. Mol Cancer 2011;10:138.
OncoTargets and Therapy

\section{Publish your work in this journal}

OncoTargets and Therapy is an international, peer-reviewed, open access journal focusing on the pathological basis of all cancers, potential targets for therapy and treatment protocols employed to improve the management of cancer patients. The journal also focuses on the impact of management programs and new therapeutic agents and protocols on

\section{Dovepress}

patient perspectives such as quality of life, adherence and satisfaction The manuscript management system is completely online and includes a very quick and fair peer-review system, which is all easy to use. Visit http://www.dovepress.com/testimonials.php to read real quotes from published authors. 